\title{
Replicating Pulsar Behaviour to Create a Future Space Propulsor
}

\author{
P. A. Murad \\ Morningstar Applied Physics Inc., LLC, Vienna, VA 22182
}

\begin{abstract}
Binary pulsars demonstrate unusual gravitational behaviour that is a careful balance between the type of orb it, companion star and neutron star weights, and neutron star spin rate. So me binary pulsars are be lieved to have the same weight for both stars moving in a highly elliptical orbit while some binaries with vast weight differences, are in near-circular orb its. This is counterintuitive and may lie outside of the conventional wisdom. Each neutron star in these binary systems spins at different rates, imply ing that rotation per Winterberg's conjecture may induce a repulsive gravitational source analogous to generating negative matter that can negate inertial effects. Moreover, swirling jets leaving black holes imp ly that these jets may move either at greater than light speed or also become a repulsive gravitational source. Interactions between the pulsar binary's two bodies and behaviour of two of Jupiter's moons may also validate notions from Je fimenko, who claims that gravity induces angular momentum. If true, these findings show that a significant relationship exists between gravity and angular momentum as well as suggest angular momentum may result in linear momentum after going through an intermediate step to produce repulsive gravitation. Furthermo re, these capabilities may be the pre requisites to devise future star ship propulsion drives to explore the cosmos.
\end{abstract}

Keywords Binary Pulsar, Neutron Star, Asteroid, Gravity, Jefimenko, Rotation, Angular Momentu m, Trajectories

\section{Introduction}

One wonders how revealing nature's secrets can provide valuable insights to develop an advanced propulsion system. This is part of an on-going investigation $[1,2]$ that examines the premise that angular momentum may be converted into linear momentum with the notion of eventually creating a propulsive drive. Basically, this premise may initially appear outrageous but a car, a bicycle, locomotive or a propeller-driven airplane essentially converts angular momentum into linear momentum. The ground transportation capability does so by using the ground to achieve traction while the airplane goes through an intermediate process where the propeller induces a force by increasing the linear momentum of the surrounding air to create forward motion. What would be the mechanis m or intermediate step for creating such traction in space viable for a space ship drive?

In a previous paper[1], the author examined this idea concerning angular mo mentum by exp loiting ano ma lous b e haviour associated with both neutron stars and black holes. Jeong[3] suggests that jets leave both types of celestial bodies. The problem may be straightforward for a neutron

* Corresponding author:

Ufoguypaul@yahoo.com (P. A. Murad)

Published online at http://journal.sapub.org/ astronomy

Copyright (C) 2012 Scientific \& Academic Publishing. All Rights Reserved star because there are no physical constraints regarding the speed of light. However, the is sue for a black hole is far more complicated. Murad[1,4,5] discussed the gravitational anomalies involving aspects of angular momentum that Jefimenko identified that could not be reproduced by Newtonian gravitation. The latter suggests gravity is only an attractive force. Moreover, Jefimenko[6] created his own gravitational model that includes a gravitational field and a weaker cogravitational field. He claims that gravity was not only an attractive force but also induces angular mo mentum. At relativistic speeds, Jefimenko suggests that the gravitational field acts like an electric field while the cogravitational field acts, based upon Heaviside's notions, like magnetism. These effects alter the law such that gravity is not only a function of distance but can be a function of both distance and velocity. Binary pulsars were examined to see if they could offer any insights coupling this notion that gravity could be an attractive force and source of angular momentum with the implication that rotation could also induce a repulsive gravitational source. It is suggested in Murad[1, 4, 5, 7] that older and more established binary pulsars, which normally consist of a neutron star and a companion, usually move in a single elliptical trajectory. For this Keplerian motion to occur, analys is implies both bodies in the binary must be of comparable weight. This appears to be coincidental that both bodies just happen to possess the same weight fraction. How can this occur if the celestial bodies moving throughout the cosmos have a random mass 
distribution? Something more fundamental is at work here! Some binary pulsars do not meet these requirements and their trajectory parameters degenerate to collapse two separate elliptical orb its into a single orbit. It is suggested the loss in the pulsar's trajectory energy results in generating a large spectrum of gravitational energy in the form of gravitational waves. However, without a gravity wave detector, it is difficult to experimentally characterize such waves except only theoretically.

\section{Objectives}

The objective is to find several binary pulsars where the neutron star and companion have similar weights. However, if the neutron stars have significantly different rotation rates, this implies that different repulsive gravitation source strengths exist in each of these binaries. Murad[1] provides a formula that indicates a repulsive gravitational source may cancel some of the inertial gravitational source thereby making both the neutron star and its companion of comparable weight. This supports Jefimenko's conjecture that gravity is attractive and a source for angular momentum. The intention is to examine this possibility further and evaluate any field propulsion implications.

\section{Methods}

There are several is sues regarding mechanis ms or physical phenomenon that have propulsion implications. Three separate issues will be discussed to include:

- Conversion of angular momentum into linear momentu $\mathrm{m}$,

- The possibility of Black hole jets creating repulsive gravitation, and

- Dynamics for the formation of pulsars.

Each will be examined separately with a combined discussion and analysis with the overall objective of uncovering propulsion implications that would allow mankind to explore the near- and the far-abroad. Their interre lationships will be summarized in the Conclusions.

\subsection{Angular Momentum Conversion}

The author implies in a previous paper[2] that angular momentum could be transferred into linear momentum especially if nonlinear effects are realized; say for example, in Einstein's field equations. Usually these problems are evaluated using a linearized or a vector version of the gravity tensor. This means that only main diagonal terms exist and off-diagonal terms are ignored. If an off-diagonal element does exist, effects from one conservation equation would stream into another and the same would hold for different space-time continuums. The latter effects would be difficult to discern especially since we only live in a four dimensional space-time with assumed linearized spatial and temporal dimensions. It would be meaningless and beyond our current intellectual grasp to relate these to unseen dimensions that span an expanded space-time continuum. In fact the author discusses where a space-time could exist having one dimension that includes linear time while a fifth dimension includes exponential time. Such events are hard to visualize or sense. In such a continuum, you could tell time using both a linear and an exponential timepiece and both answers may be correct. In fact we can do that in our current space-time continuum where memory exponentially reaches a past time that we desire to recall and bring these events to clear focus in the present. Unfortunately only our imagination could predict a future event in our world but such a prediction would be fraught with huge uncertainties.

\subsection{Jefimenko's Conjecture}

Jefimenko's notions[e.g., 7] are contrary to Newtonian gravitation, which is considered only as an attractive force. Is there any semblance of proof that this is true? Two moons of Jupiter, Himalia and Elara are probably more recently captured asteroids that have not yet had sufficient time in orbit to synchronize their period and rotation. Himalia is the tenth known satellite of Jupiter. As the brightest of Jupiter's outer satellites, Himalia, was captured by the Cassini mis sion, for the first time, in a series of narrow angle images taken on December 19, 2000. This was from a distance of 4.4 million kilometers during the brief period when thrusters instead of reaction wheels stabilized Cassini's attitude. It is likely that Himalia is not spherical but is believed to be an irregularly shaped asteroid.

Himalia is at a distance of $11,480,000 \mathrm{~km}$ from Jupiter's surface and rotates every 0.4 days but requires 250.6 days to complete a revolution around Jupiter. So, an observer on Jupiter would certainly be exposed to all sides of Himalia, and similarly with Elara. Very little is known about Elara that is at a distance of $11,737,000 \mathrm{~km}$ from Jupiter's surface and rotates every .5 days with an orbitalperiod of 259.6 days. Comparing these numbers, as the orbital period increases, the rotational period increases. Differences are due to orbital eccentricity for Himalia of .1580 and the orbital eccentricity for Elara of . 2072. Most of the asteroids that have been closely observed seem to be rotating. Unless the capture mechanis m involved a collision, it is hard to know how the angular mo mentum would change by the capture process. It is possible that these two moons were rotating before they were captured and maintained that rotation rate while being captured. After capture, tidal effects, or whatever process that creates synchronization, would slowly reduce the rotation rate trans ferring angular mo mentum to Jupiter. Light can carry angular momentum, so heat from tidal friction could radiate some of the angular momentum into space. In other words, two satellites with almost equal weight but different geometric shapes tend to orbit Jupiter at similar distances and have similar rotation rates that imply Jupiter's gravitation is inducing angular momentum. This has to be more than coincidental.

\subsection{Speed of Jets from a Black Hole}


A black hole may be a collapsed star where the forces of gravity are so large that even light does not escape. This means that everything that moves at or less than the speed of light will remain within the black hole. This also includes magnetic and electric fields. If a jet leaves the black hole, it must either move at greater than the speed of light or by some other unknown mechanis $\mathrm{m}$. If greater than light speed, then the jets are clearly evidence of naturally occurring hyper-light phenomenon. Thus it would be beneficial to find a black hole that is not rotating with no accretion disk but with a jet. Furthermore, the next question is to try and understand how much faster than the speed oflight does such a jet move at and is there some limit by Mother Nature for the jet velocity?

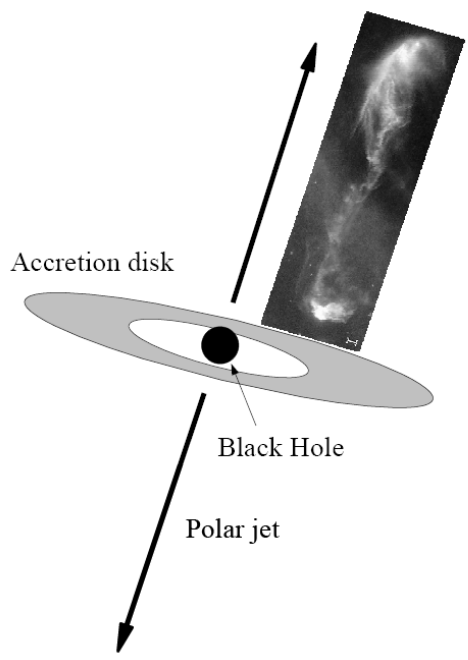

Figure 1. Scheme of a Herbig-Haro object HH47 andthe collimated jets of partially ionized gas claimed as belonging to the accretion disk, taken by the Hubble Space Telescope

One may argue that the jet consists of debris possessing inertial mass from the surrounding accretion disk. Some black holes may have a jet but no accretion disk. This is a strong possibility; however, if from the black hole its elf, the jet consists of a spiral that is moving outward along its ejection axis as well as rotating about the ejection axis. Unfortunately, there is no direct means to measure the rotation rate of a black hole or for that matter, the jet rotation rate. Such rotation can be approximated only indirectly by examining effects within the surrounding environment. However, it is still quite difficult to accurately perform such a task. This is not the case for a neutron star where the beam of radiation would sweep over the Earth for detection due to the lighthouse effect. Neutron stars rotate at speeds that can range from 10 to 600 times a second. These stars have masses that could be far larger than our sun rotating at these large rates. Jeong[3] implies that the jet from a black hole or a neutron star may be forced outward by a repulsive gravitational source. It is hypothesized that the spiral motion within the jet can create a repulsive gravitational source based upon an analysis of a Magnetar by Winterberg[1, 2]. Here we can assume that the jet swirls at such a rotation rate that a repulsive gravitational source may result. Moreover, convergence of cloud material could be nothing more than gas dynamic shocks coalescing due to deceleration of the jet matter caused by the original backward gravitational pull of the black hole.

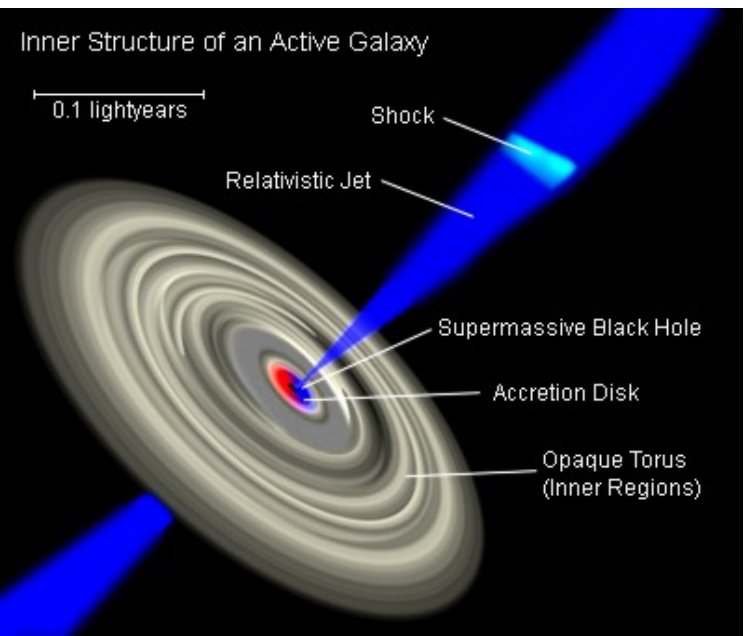

Figure 2. Some details may indicate a gas dynamic shock may appear in the jet as suggested in this artist rendition

Under the gravitational force magnitude interpretation, an object with negative mass would repel ordinary matter, and could be used to produce an anti-gravity effect. Alternatively, depending on the mechanis $m$ assumed to underlie the gravitational force, it may seem reasonable to postulate a material that shields against gravity or otherwise interferes with a gravitational force. A Magnetar is a neutron star with an extremely strong magnetic field generated by the convection of hot nuclear matter produced as a consequence of nuclear reactions. Winterberg[8] looks at a laboratory analogue of a geodynamo or Magnetar that involves a rapidly rotating liquid metal,

$$
\nabla \cdot \bar{g}=-4 \pi G \rho=2 \omega^{2}, \quad \text { where } \rho=-\frac{\omega^{2}}{2 \pi G} .
$$

According to Winterberg, the source term $\rho$ is negative for a repulsive mass density. If a gyroscope is placed at $45^{\circ}$ on a table and let go, the gyroscope falls. However, if the rotor is spinning, it is capable of remaining aligned at this initial angular orientation. As the rotor speed decays, the gyroscope starts to precess rotating in a circumferential direction. When the rotation drops below a certain limit, the gyroscope falls to the tabletop. The conventional wisdom suggests angular momentum couples explain this effect. An alternative solution is that the rotation may induce a repulsive gravitational source that levitates the gyroscope according to this equation. Moreover, one could classify this as an 'Inverse Corio lis effect'. Another way of considering this is that a gravitational field would repulse negative mass. Such a source can be considered as negative matter[9].

\subsection{Puls ars}

A pulsar or neutron star is the collapsed core of a massive star that has ended its life in a supernova explosion. Weighing more than our Sun, the star collapses because of 
the gravitational forces to become only about 20 kilometers across. As the star collapses, angular momentum conservation indicates the rotation rate should increase. The collapsed star has an intense magnetic field that may not align with the rotation axis. These incredibly dense objects produce beams of radio waves, as well as X-rays, which sweep across the sky like a lighthouse, often hundreds of times a second. Radio telescopes receive a regular train of these pulses as the beam repeatedly crosses the Earth so the objects are observed as a pulsating radio signal. Puls ars make exceptional clocks, which enable a number of unique astronomical experiments. Some very old pulsars, which can reach speeds of over 600 rotations per second, rotate so smoothly that they may "keep time" more accurately than the best atomic clocks here on Earth.

One can postulate the formation of a binary pulsar in that the strong gravitational attraction would pull another celestial body within the vicinity of the Neutron star. For binaries, there can be a flow of material into the neutron star from a companion star. However, analys is to date tends to suggest that the weight of a companion and the attracting neutron star are comparable. This is unusual because the weight differences should be random and would impact the types of orbits about each body. If one is to accept Jefimenko notion that gravity induces angular momentum, then there should be an effect that impacts the rotation rates of both the primary and companion star. Unfortunately, there is no technique available to determine the rotation rate of the companion star in a pulsar binary system. Moreover, regarding Winterberg[8] where rotation creates a repulsive gravitational force, the two bodies may simulate a binary system where each body appears to have comparable weight by balancing the rotation rate of the neutron star. If gravity changes angular momentum, then the companion will affect the rotation rate of the neutron star with a careful balance occurring between rotation rates and the subsequent orbits of both bodies.

If $\mathrm{W}$ interberg is correct, then the inertial mass of the neutron star has to be greater than the companion star to compensate for the loss of gravitation due to spin. In other words the neutron star should weigh more and the value depends upon the rotation speed. Stars at $600 \mathrm{rps}$ would produce more compensation compared to a neutron star that rotates at about $10 \mathrm{rps}$. The neutron star source term is greater by:

$$
\rho_{\mathrm{p}}=\rho_{\mathrm{c}}+\frac{\omega_{\mathrm{p}}^{2}}{2 \pi \mathrm{G}} \text { and: } \mathrm{W}_{\mathrm{p}} \approx\left[1+\frac{\omega_{\mathrm{p}}^{2}}{2 \pi \mathrm{G} \rho_{\mathrm{c}}}\right] \mathrm{W}_{\mathrm{c}}
$$

The subscript ' $p$ ' is for the neutron star while the ' $c$ ' is the value for the companion star. The neutron star may be located at the epicenter of the elliptical trajectory and the companion star essentially orbits about the neutron star. Such a trajectory is more conventional for the two-body problem of space mechanics as one possibility. A second possibility is that both bodies travel in an elliptical orbit based upon their mutual attraction for each other. Thus, the suggestion is that we may have underestimated the weight of celestial bodies and the additional weight may negate the need for finding additional mass thereby competing with such hypotheses as dark matter to explain anomalous phenomenon[10]. Differences in masses may dictate the neutron star spin rate as an artifact of a natural gravitational process where gravity is not only an attractive force but induces angular momentum per Jefimenko's claims. This formula suggests that the only case where a Neutron star has the same inertial weight as its companion is when there is no Neutron star rotation. No observational data for such a situation exists, which without the beam sweeping consequences produced by the lighthouse effect would essentially defy detection.

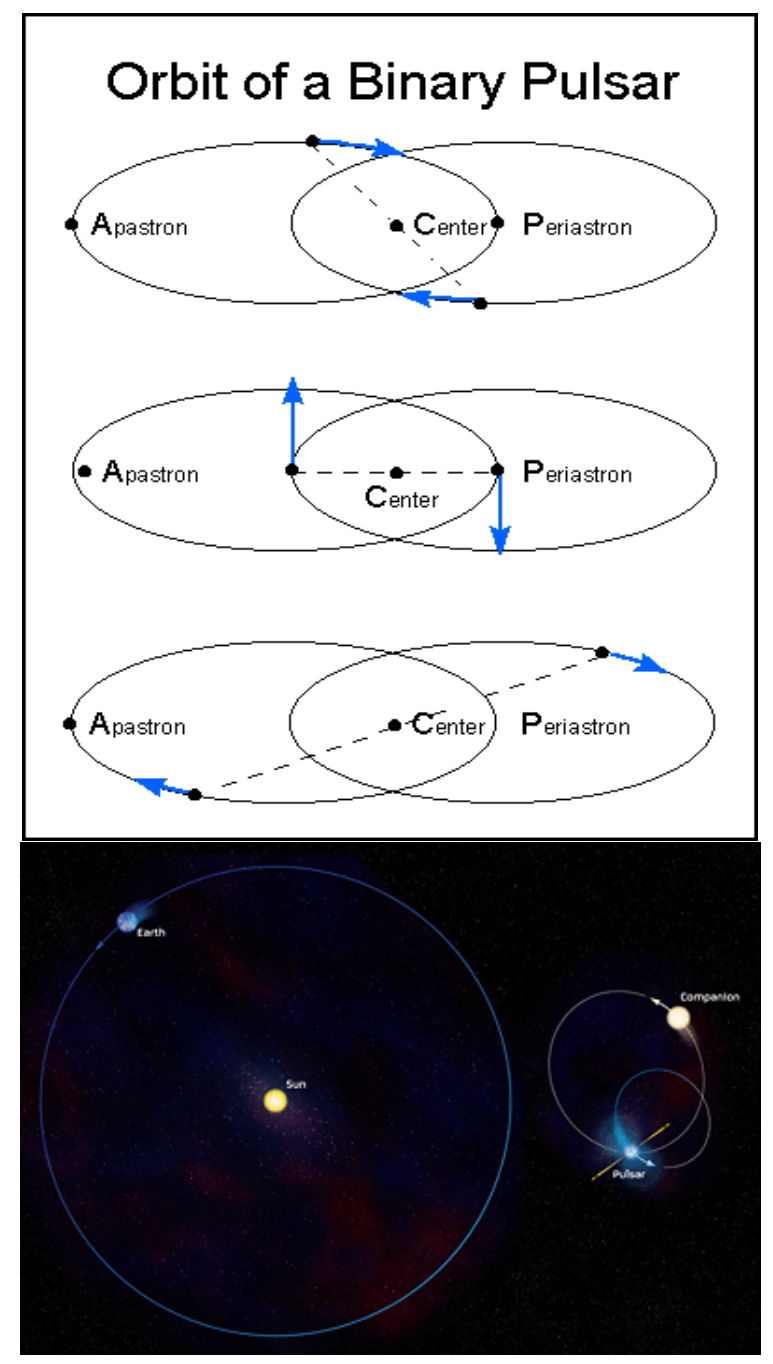

Figure 3. The orbits of the binary pulsar $1913+16$ and a comparison of the orbits of the pulsar $\mathrm{J} 1903+0327$ with its possible sun-like companion star with the orbit of the Earth around the sun[11]

Ideally, consequences of these interactions would produce a circular orbit that contains both bodies. However, this is not the case and these binary pulsars contain either a single elliptical orbit or each body would travel in its own separate elliptical orbit. Moreover, with the passage of time, mass from the stars decreases due to emission of energy in the form of X-ray, electromagnetic, or gravitational wave radiation. Thus the decreasing masses, the orbit shapes, and 
the rotation rates all have to reach certain equilibrium values to result in an elliptical orb it and with more expenditure of masses from both stars, eventually reach a circular orb it when the masses become comparable.

\section{Results}

As the binary pulsar evolves for two bodies, it goes from two erratic elliptical orbits to collapse into a single orbit. With time, considering that the mass decreases in the Neutron star, the single elliptical orbit evolves into a single circular orbit. If this is the correct evolution, the propulsion implications are obvious. If true, then clearly rotation or angular mo mentum has a direct relationship with gravity and Jefimenko was right in his conjecture. Thus, there is a direct connection between angular and linear momentum. Moreover, Winterberg would also be correct in his notion that rotation can induce a repulsive gravitational effect and this also has propulsion implications.

Table 1 has some interesting data. Each mass is in solar masses. Earlier it was suggested the Neutron star should weigh more than the companion star. Differences shown here may indicate that in these binary systems, the companion star may also be a Neutron star; however, without the lighthouse effect, detection is denied. Data was taken to evaluate several binary pulsars to establish some insights regarding the size of the primary body, a Neutron star, and its companion. This data shown has some uncertainty associated with it by virtue of the observational or radiometric data considering the large distances involved.
Moreover, the data set itself is incomplete because of the numerous sources of which most is from Wikipedia that includes claims that data flowed from peer-reviewed technical papers. Where applicable, uncertainties in the data are included. In some cases, the authors imply that the binary pulsars are moving in nearly perfect circular orb its; it shall be assumed that if not mentioned, the eccentricity will be zero. However, as shown for Pulsar 13 (PSR J1903+0327), these two bodies may traverse circular orbits but both bodies are not in the same orbit. Note that the masses of both bodies are not equal.

The is sue about mass diffe rences between the Neutron star and the companion within the binary system is a major concern. Pulsar 5 (PSR B1534+12) and Pulsar 9 (PSR B2127+11C) are situations where the Neutron star and companion star are very close to the same weight. However, these are in elliptical orb its with eccentricity of .2736 and .68 respectively. The latter is considerable. However, note also that the weights for each binary star are comparable. The only difference for these binaries is the rotation rates of the Neutron stars. It is assumed that the orbits of both bodies in each of the binaries are in the same orbit. The point is that if the weights of the Neutron star and the companion are close to each other, then they should be in a single circular orb it. If a new capture, the orbit should decay to one with slight eccentricity. This is not the situation here by any stretch of the imagination. One could assume that the rotation rate of the Neutron star is creating an imbalance that creates such orbital kinematics.

Table 1. Pulsar Information

\begin{tabular}{|c|c|c|c|c|c|c|}
\hline Pulsar & Eccen tricity & $\mathbf{P}(\mathbf{m s})$ & $\mathbf{M}_{\text {primary }}$ & $\mathbf{M}_{\text {comp }}$ & Rotation & Orbit \\
\hline & $\epsilon$ & & & & Rate & Period \\
\hline 1. J1141-6545 & 0.17 & 393.93 & $1.30 \pm .02$ & $.986 \pm .02$ & $2.538 \mathrm{~Hz}$ & $4.77 \mathrm{hr}$ \\
\hline 2. $\mathrm{B} 2303+46$ & 0.66 & 1066 & $1.40(?)$ & 1.24 & $0.93 \mathrm{~Hz}$ & $296.2 \mathrm{hr}$ \\
\hline 3. PSR J0437-4715 & 0.00 & 5.75 & 1.8 & .25 & $173.7 \mathrm{~Hz}$ & $5.75 \mathrm{~d}$ \\
\hline $\begin{array}{c}\text { 4. PSR } \\
\text { J1518+4904 }\end{array}$ & 0.25 & 40.9 & 1.75 & $0.9-2.7$ & $24.44 \mathrm{~Hz}$ & $8.6 \mathrm{~d}$ \\
\hline 5. PSR B1534+12 & 0.2736 & 37.0 & 1.339 & 1.339 & $26.38 \mathrm{~Hz}$ & $.4207 d$ \\
\hline 6. PSR J181 1-1736 & 0.828 & 104.2 & $2.6 \pm .9$ & 0.7 & $9.59 \mathrm{~Hz}$ & $18.8 \mathrm{~d}$ \\
\hline 7. PSR B1820-11 & 0.79 & 279.8 & ---- & ---- & $3.58 \mathrm{~Hz}$ & 357.8 \\
\hline 8. PSR B1913+16 & 0.61713 & 37.904 & $1.4414 \pm .0002$ & $1.3867 \pm .0002$ & $26.3 \mathrm{~Hz}$ & .3229 \\
\hline $\begin{array}{c}\text { 9. PSR } \\
\text { B2 } 127+11 \mathrm{C}\end{array}$ & 0.68 & 30.5 & $1.34 \pm .2$ & $1.365 \pm .2$ & $32.7 \mathrm{~Hz}$ & .3 \\
\hline \multicolumn{7}{|l|}{ 10. PSR J0737-3039 } \\
\hline Pulsar A & ---- & 22.7 & 1.337 & & $44.04 \mathrm{~Hz}$ & $50 \mathrm{sec}$ \\
\hline Pulsar B & ---- & $2.77 \mathrm{sec}$ & 1.250 & & $0.36 \mathrm{~Hz}$ & $.3667 \mathrm{~s}$ \\
\hline 11. PSR B1534-12 & 0.2736 & --- & 2.67838 & 1.339 & ---- & $.4207 \mathrm{~d}$ \\
\hline $\begin{array}{l}\text { 12. PSR } \\
\text { J1748-2446ad }\end{array}$ & 0.00 & 1.39 & $\sim 2.0$ & .14 & $716 \mathrm{~Hz}$ & $26 \mathrm{Hr}$ \\
\hline 13. PSR J1 903+0327 & 0.437 & 2.15 & 1.74 & 1.0 & $465 \mathrm{~Hz}$ & $95.17 \mathrm{~d}$ \\
\hline 14. PSR B1937+21 & ----- & 1.5577 & ----- & ----- & $641.9 \mathrm{~Hz}$ & ---- \\
\hline
\end{tabular}


There is a contrasting situation as well. This is with Pulsar 3 (PSR J0437-4715)[12, 13] and Pulsar 12 (PSR $\mathrm{J} 1748-2466 \mathrm{ad}$ ) where the eccentricity is zero and it is assumed the Neutron star and companion are in the same circular orbit unlike the situation shown for Pulsar 13 $(\mathrm{J} 1903+0327)$. Optical observations from several references indicate that the binary companion of Pulsar 3 (PSR J0437-4715) is most likely a low-mass helium white dwarf. The pulsar is about 1.8 solar mass and the companion is only about 0.25 solar masses. The pair revolve a round each other every 5.75 days in nearly perfect circular orbits. This would imply that to reside in a circular orbit, both the weight of the Neutron star and the companion star should be equal. However, as shown, the mass of the Neutron star is almost an order of magnitude greater than that of the companion star. Moreover, the rotation rates are quite close as well. This implies that the rotation rate may potentially decrease the inertial mass quite a considerable amount to match the inertial mass of the companion star. If true, this suggests that rotation effects may create a situation that results in a component having a repulsive gravitational source.

If these masses for the binaries are correct, this is a significant finding for creating a future space propulsion capability. In other words, our spacecraft may have to replicate and duplicate the characteristics of a Neutron star in a binary pulsar if we are to become a space-faring civilization.

\section{Conclusions}

We may have accidentally discovered the Holy Grail of space travel. Converting angular mo mentum to create linear momentum can occur in space when it goes through an intermediate step of inducing a gravitational repulsive source. This is demonstrated by rotating swirls of inertial jets to produce a repulsive source that is strong enough to leave the gravitational field of a Black Hole. This will create a forward acceleration or enough linear momentum to leave the Black Hole. Moreover, binary pulsars appear to enjoy a careful balancing act between the primary and companion star whose masses can be significantly different. These stars use gravitational angular momentum effects to determine the Neutron star's rotation rate. Based upon these characteristics, subsequent orbits will initially be separate elliptical orbits that slowly evolve into a single elliptical orbit. Thus when the 'virtual' masses of both stars are nearly equal, either by consuming Neutron star mass to produce energy over time or change the star's rotation rate, the orbit of the stars should circularize into a single circular orbit. Now the hard part, 'How do we convert this knowledge, if true, into hardware for a future space drive to take mankind to new star systems?'

\section{ACKNOWLEDGMENTS}

The author wishes to acknowledge information received from John Cole, formerly of NASA Marshall Spaceflight
Center, regard ing the Moons of Jupiter. The author wishes to also thank Mr. Dana Sweet for helpful criticis ms.

\section{REFERENCES}

[1] Murad, P. A., An Anzatz about Gravity, Cosmology, and the Pioneer Anomaly, in Proceedings of Space, Propulsion and Energy Sciences International Forum, edited by G.A. Robertson, AIP Conference Proceedings 1208, Melville, New York, 2010.

[2] Murad, P. A., The Challen ges of Developing the Technology for A Realistic Starship Propulsor, presented at the 48th AIAA Aerospace Sciences Meeting, AIAA Paper 2010-1609, 2010.

[3] Jeong, E. J., An Isolated Gravitational Dipole Moment Placed at the Center of the Two Mass Pole Model Universe, arxiv.org, 1997.

[4] Murad, P. A., Warp-Drives, The Dreams and Realities, Part I, A Problem Statement and Insights, in the proceedings of the Space Technology and Applications International Forum (STAIF-05), edited by M. S. El-Genk, AIP Conference Proceedings 746, Melville, New York, 2005, pp. 1256-1263.

[5] Murad, P. A., Warp-Drives, The Dreams and Realities, Part II, Potential Solutions, in the proceedings of the Space Technology and Applications International Forum (STAIF-05), edited by M. S. El-Genk, AIP Conference Proceedings 746, Melville, New York, 2005 p. 1411-1418.

[6] Jefimenko, O. D., Gravitation and Cogravitation, Electret Scientific Company Star City, 2006.

[7] Murad, P. A., An Alternative Explanation of the Binary Pulsar PSR 1913+16, in Proceedings of Space, Propulsion and Energy Sciences International Forum, edited by G.A. Robertson, AIP Conference Proceedings 1103, Melville, New York, 2009.

[8] Winterberg, F.: Thermonuclear Dynamo inside an Alfven Black Hole, University of Nevada, Reno, 2006.

[9] Brandenburg, J. E. and Kline, J. F., Application of the GEM theory of Gravity-Electro-Manetism Unification to the Problem of Controlled Gravity, Theory and Experiment, presented at the 34th Joint Propulsion Conference \& Exhibit, AIAA 98-3137, 1998.

[10] Murad, P. A., Gravity Laws and Gravitational Wave Phenomenon, Is There a Need for Dark Mass or Dark Energy? Presented at the AIAA/ASME/SAE/ASEE Joint Propulsion Conference, AIAA 2008-5123, 2008.

[11] Dunham, W., Astronomers Baffled by Weird, Fast-Spinning Pulsar, Reuters, Washington, DC, 2008.

[12] Bell, J. F., Bailes, M., Bessell, M. S., Optical detection of the companion of the millisecond Pulsar J0437 - 471, Nature 1993 364(6438): 603-605.

[13] Verbiest, J. P. W., Bailes, M., van Straten, W., Hobbs, G. B., Edwards, R. T., Manchester, R. N., Bhat, N. D. R., Sarkissian, J. M., Jacoby, B. A., Kulkarni, S. R., Precision Timing of PSR J0437-4715, An Accurate Pulsar Distance, a High Pulsar Mass, and a Limit on the Variation of Newton's Gravitational Constant, The Astrophy sical Journal (2008) 679(1): 675-680. 